\title{
Epilepsien - Behandlungsmöglichkeiten ausschöpfen
}

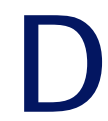
as Schwerpunktthema dieses Heftes behandelt mit den Epilepsien eine der häufigsten chronischen Erkrankungen in der Neurologie. Bis zu 1\% der Bevölkerung leiden an einer Epilepsie und 5\% haben einmal in ihrem Leben einen epileptischen Anfall erlitten.

Die Diagnostik und die Therapie der Epilepsien haben im letzten Jahrzehnt einen enormen Fortschritt erlangt, so dass in Abhängigkeit von dem Syndrom bis zu 90\% der Patienten anfallsfrei werden können. Diagnostisch hat insbesondere die Verbesserung der Bildgebung die Epilepsiechirurgie beeinflusst. Die Entwicklung neuer Antiepileptika mit neuen Wirkmechanismen und Präparaten, die nicht nur antikonvulsiv, sondern auch antiepileptogen wirken, hat zu einem Qualitätssprung in der medikamentösen Behandlung der Epilepsien geführt.

Allerdings werden im klinischen Alttag die Möglichkeiten nicht ausgeschöpft. Besorgnis macht das Ergebnis einer Untersuchung an unserem Epilepsiezentrum, das zeigt, dass gut die Hälfte der Patienten, die nach durchschnittlich 15-jährigem Krankheitsverlauf zugewiesen werden, noch eine falsche Syndromdiagnose hat und daraus resultierend häufig eine falsche Therapieentscheidung.

Woran liegt das? Es ist nicht das fehlende Wissen über differenzierte neue Wirkmechanismen von Antiepileptika, sondern es liegt an Basisdingen epileptologischer Arbeit. Beim Erstkontakt nach einem epileptischen Anfall werden häufig die Weichen falsch gestellt. Es besteht eine Unsicherheit in der Differenzierung der Syndrome, in der Unterscheidung von benignen und malignen Epilepsieverläufen, und es besteht Unklarheit, wann und unter welchen Gesichtspunkten eine Beratung zu einem epilepsiechirurgischen Eingriff erfolgen soll.

So haben wir uns bei diesem Schwerpunkt auf Basisthemen konzentriert, aber bei dem zunehmend wichtig werdenden Thema der Pharmakoresistenz auch Aspekte der Grundlagenforschung aufgegriffen. Das Verständnis der Pharmakoresistenz, zu dem in den nächsten Jahren die Pharmakogenetik wesentliches beitragen wird, wird wichtige Grundlage sein für die Weiterentwicklung neuer Therapiekonzepte.

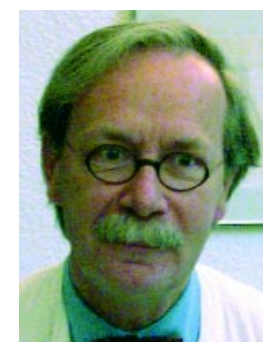

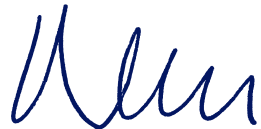

Prof. Dr. H.-J. Meencke, Berlin 